\title{
The relationship of serum asymmetric dimethylarginine concentrations and lung involvement in patients with COVID-19 infection
}

\author{
Nadir Emlek ${ }^{1}$ Ahmet Seyda Yilmaz ${ }^{1}$ Elif Ergul ${ }^{1}$, Hasan Gundogdu², Medeni Arpa ${ }^{3}$, \\ Haldun Koc', (iD) Muhammet Ozturk ${ }^{1}$, Cihan Aydin ${ }^{4}$
}

${ }^{1}$ Department of Cardiology, Recep Tayyip Erdogan University, Faculty of Medicine, Rize, Turkey

${ }^{2}$ Department of Radiology, Recep Tayyip Erdogan University, Faculty of Medicine, Rize, Turkey

${ }^{3}$ Department of Biochemistry, Recep Tayyip Erdogan University, Faculty of Medicine, Turkey

${ }^{4}$ Department of Cardiology, Namık Kemal University, Faculty of Medicine, Tekirdă̆, Turkey

\section{ABSTRACT}

Aim: COVID-19 infections the tissue through angiotensin converting enzyme 2 receptor, which is also expressed on endothelial cells. Endothelial dysfunction may be associated with lung involvement. Asymmetric dimethylarginine (ADMA) is an indirect marker of endothelial dysfunction. The aim of our study was to evaluate ADMA concentrations and to identify its association with lung involvement in patients with COVID19 disease.

Methods: We included 42 patients with COVID-19 infection and lung involvement (Group 1). Forty-two age and sex matched patients without pneumonia acted as the control group (Group 2). All patients gave blood samples for ADMA at the $1^{\text {st }}$ month control visit after discharge. We compared C-reactive protein (CRP) and ADMA concentrations in addition to routine biochemical parameters between groups.

Results: Patients with lung involvement had higher admission glucose, CRP, and ADMA concentrations, and displayed lower hemoglobin concentration and lymphocyte count compared to patients without lung involvement. Although patients with lung involvement had higher ADMA concentrations with respect to those without; plasma ADMA levels were also higher than normal values in control group. Multivariate analysis identified log CRP concentration $(O R=3.047,95 \% C I=1.881-5.023, p<0.001)$ as the independent predictor for lung involvement. And, there was a correlation between ADMA and CRP ( $r: 0.318, p: 0.003)$.

Conclusion: We revealed elevated ADMA concentrations as the surrogate of endothelial dysfunction in COVID-19 patients whether they have pneumonia or not.

Key words: COVID-19, pneumonia, endothelial dysfunction, asymmetric dimethylarginine, CRP.

$\triangle$ Dr. Nadir Emlek

Department of Cardiology, Recep Tayyip Erdogan

University, Faculty of Medicine, Rize, Turkey

E-mail: emleknadir53@gmail.com

Received: 2021-05-22 / Revisions: 2021-07-03

Accepted: 2021-08-17 / Published online: 2021-10-01

\section{Introduction}

SARS-CoV-2 infection, also nominated as Coronaviral Disease 2019 (COVID-19), was characterized as a pandemic since March 2020 by World Heart Organization [1]. The number of cases and mortality has increased gravely ever since [2]. Although the majority of patients tend to be well or asymptomatic, $14 \%$ experience severe, and $5 \%$ suffer critical disease with lung involvement [3].

Interestingly several cardiovascular comorbidities have been identified to be 
associated with a higher rate of pulmonary complications.

COVID-19 infects the tissue thorough angiotensin converting enzyme 2 receptor. This receptor is abundantly expressed in endothelial cells besides lung, heart, kidney and bowel tissue [4]. The infection of endothelial lining may induce endothelial dysfunction, which may be in part responsible for multi-systemic involvement. Endothelial dysfunction can be assessed by several invasive or non-invasive methods [5]. Nitric oxide (NO), synthesized by the endothelial nitric oxide synthase, is a multifunctional molecule involved in vasodilation, and the immune system. NO has additional activity in cytokine secretion, nonspecific immunity, inhibition of viral replication, and transplant rejection [6,7]. Asymmetric dimethylarginine (ADMA) is an inhibitor of endogenous nitric oxide synthase. Since serum ADMA concentration is inversely related with nitric oxide synthesis, ADMA concentration may be an indirect marker of endothelial dysfunction. Moreover, ADMA is non-invasive, cheap and a practical measure for detecting endothelial dysfunction.

As we have witnessed a global pandemic in the past months, identification co-morbidities and predictors associated with more frequent lung involvement and higher mortality is essential. We hypothesized that ADMA concentrations reflecting endothelial dysfunction might be related to lung involvement in this specific patient group. So, this study planned to investigate ADMA concentrations in patients with COVID-19 infection

\section{Materials and methods}

\section{Study Design \& Demographics}

Our study, prospective, and observational cross-sectional cohort in-design, included consecutive 42 patients with COVID-19 infection and lung involvement (Group 1). Age and sex matched consecutive 42 COVID-19 patients without pneumonia acted as the control group (Group 2). Assuming an alpha of 0.05, a power of 0.80 , and $30 \%$ potential change from baseline value in terms of pulmonary involvement consistent with previous reports, the estimated sample size was at least 40 patients in total.

All patients were informed about the study and their written consent was given. The research was realized in accordance with the principles set by the Declaration of Helsinki, the International Good Clinical Practice guidelines and all applicable legal requirements. The study protocol was approved with registration number of 2020/164 by Recep Tayyip Erdoğan University School of Medicine Ethics Committee.

Patients who did not have any lung pathology at onset and got lung involvement in the followup period were included in the first group. Diagnosis of COVID-19 was proven using polymerase chain reaction (PCR) method and/or computed tomography (CT). Medication history including previous and novel medications throughout hospitalization were recorded.

We excluded patients with immunosuppressive treatment, renal or hepatic failure, malignancy, idiopathic pulmonary fibrosis, and patients who passed away through follow-up.

Following diagnosis of COVID-19 infection by an infectious disease expert or a pulmonologist, complaints, demographic and laboratory parameters, and CT images of the patients were reviewed and recorded. Diabetes mellitus was acknowledged as the fasting blood glucose concentration > $126 \mathrm{mg} / \mathrm{dL}$ or any blood glucose measurement $>200 \mathrm{mg} / \mathrm{dL}$ or use of anti-diabetic medication. Hypertension was accepted as blood pressure above 130/85 
mmHg or use of anti-hypertensive drugs. Those who smoked regularly in the last 6 months were denoted as smokers.

\section{Biochemical analysis}

Blood samples were taken for ADMA analysis using antecubital veins at the first month control of recovered patients and stored in a $80^{\circ} \mathrm{C}$ refrigerator after centrifugation. Human ADMA Elisa kit (Cloud-Clone Corp., China) was used to detect serum ADMA levels. While assay range is indicated between $12.35 \mathrm{ng} / \mathrm{mL}$ and $1000 \mathrm{ng} / \mathrm{mL}$, the intraassay and interassay coefficient of variations (CV) were $<10 \%$, $<12 \%$ respectively, according to the manufacturer. The sensitivity of the assay was stated as $4.99 \mathrm{ng} / \mathrm{mL}$ by manufacturer.

$\mathrm{C}$ reactive protein (CRP) levels were quantitated using immunoturbidimetric assay on Beckman Coulter AU5800 autoanalyzer (Beckman Coulter Diagnostics, USA). While the assay range for CRP was below $5 \mathrm{mg} / \mathrm{L}$, the within run and total precision are less than $5 \%$ $\mathrm{CV}$, according to the manufacturer.

\section{Computed tomography}

Thorax CT acquisitions were performed by Alexion 16 detector CT (Toshiba Medical Systems, Japan) machine in the supine position during breath-holding following deep inspiration from lung apices to umbilicus without non-ionic contrast, using the parameters of $120 \mathrm{kV}, 125 \mathrm{~mA}, 16 \times 1.5 \mathrm{~mm}$ collimation and $3 \mathrm{~mm}$ thickness, $512 \times 512$ matrix. A specialized thoracic radiologist assessed axial views at parenchyma window (1500 HU, -600 HU). The images were transferred to a workstation to evaluate typical or atypical COVID-19 disease signs. The patients were staged according to Radiological Society North America (RSNA) criteria using CT images of patients during hospitalization. Typical appearance includes highly specific imaging features, indeterminate appearance denotes non-specific features and atypical appearance implies uncommonly or not reported features of COVID-19 pneumonia. Negative for pneumonia shows no features of pneumonia. The group 1 patient mainly included patients with typical appearance, and some with indeterminate appearance. Control subjects were mostly negative for pneumonia with a few atypical appearances.

\section{Statistical analysis}

The Statistical Package for the Social Sciences 20.0 statistical software program (SSPS Inc, Chicago, Illinois) was used for the statistical analysis. Kolmogorov Smirnov was used to check normality of continuous variables. Continuous variables were expressed as the mean \pm standard deviation. Categorical variables were presented as percentages. The normally distributed variables were compared with the Student's t-test and the variables that did not conform to a normal distribution were compared with Mann-Whitney U- test. Categorical variables were compared with chisquare or Fisher exact test. Continuous variables without normal distribution were logtransformed for univariate and multi-variate analysis. Linear and logistic regression analyses were used for the multivariate analysis of independent variables which were included if they were significantly different in the univariate analyses. All tests of significance were two-tailed. Statistical significance was defined as $p<0.05$.

\section{Results}

We included age and gender matched 42 COVID-19 patients to each group according to pneumonic involvement on CT or not. Our study included 39 female and 45 male patients with a median age of 55 (46-69). Demographic characteristics of the study group are presented in Table 1. The patients with lung involvement 
Table 1. Demographical and clinical findings of the study population.

\begin{tabular}{|c|c|c|c|}
\hline Variable & Lung Involvement (-) (n=42) & Lung Involvement $(+)(n=42)$ & $p$ \\
\hline Age (Year) & $54.2 \pm 12.8$ & $54.1 \pm 11.7$ & 0.965 \\
\hline $\begin{array}{c}\text { Gender }(\mathrm{n} / \%) \\
\text { Female } \\
\text { Male }\end{array}$ & $\begin{array}{l}19(45.2) \\
23(54.8)\end{array}$ & $\begin{array}{l}20(47.6) \\
22(52.4)\end{array}$ & $\begin{array}{l}0.829 \\
0.741\end{array}$ \\
\hline BMI $\left(\mathrm{kg} / \mathrm{m}^{2}\right)$ & $28.7 \pm 4.02$ & $27.6 \pm 2.9$ & 0.163 \\
\hline SBP (mmHg) & $124.6 \pm 12.5$ & $122.1 \pm 25.9$ & 0.558 \\
\hline $\mathrm{DBP}(\mathrm{mmHg})$ & $76.4 \pm 8.9$ & $73.3 \pm 15.1$ & 0.261 \\
\hline Heart rate $(\mathrm{bpm} / \mathrm{min})$ & $69.8 \pm 15.2$ & $74.1 \pm 21.8$ & 0.292 \\
\hline $\operatorname{LVEF}(\%)$ & $60.4 \pm 2.7$ & $59.6 \pm 3.2$ & 0.267 \\
\hline HT n\% & $21(50)$ & $22(52.4)$ & 0.830 \\
\hline Current smoking (n/\%) & $6(14.3)$ & $6(14.3)$ & 1.000 \\
\hline Fever ( C) & $14(33.3)$ & $26(61.9)$ & 0.008 \\
\hline Asymptomatic (n/\%) & $13(31.0)$ & $0(0)$ & $<0.001$ \\
\hline $\operatorname{HPL}(\mathrm{n} / \%)$ & $9(21.4)$ & 11826.4) & 0.614 \\
\hline $\operatorname{COPD}(\mathrm{n} / \%)$ & $1(2.4)$ & $1(22.4)$ & 1.000 \\
\hline Previous CAD (n/\%) & $3(7.1)$ & $6(14.3)$ & 0.296 \\
\hline B. Blocker (Adm) (n/\%) & $6(14.3)$ & $12(28.6)$ & 0.113 \\
\hline CCB (Adm) (n/\%) & $5(11.9)$ & $7(16.7)$ & 0.539 \\
\hline ACEI (Adm) (n/\%) & $4(9.5)$ & $8(19.0)$ & 0.217 \\
\hline ARB (Adm) (n/\%) & $10(23.8)$ & 8819) & 0.600 \\
\hline Statin therapy (Adm) (n/\%) & $8(19)$ & $10(23.8)$ & 0.600 \\
\hline Glucose (Adm) (mg/dL) & $107.4 \pm 22.5$ & $133.6 \pm 60$ & 0.011 \\
\hline $\mathrm{Se} \mathrm{Cr}(\mathrm{mg} / \mathrm{dL})$ & $0.86 \pm 0.33$ & $0.81 \pm 0.18$ & 0.352 \\
\hline CRP (mg/dL) & $1.72 \pm 1.39$ & $3.85 \pm 1.22$ & $<0.001$ \\
\hline DDimer ng/mL & $380(315-675)$ & $585(250-880)$ & 0.149 \\
\hline Troponin I ng/mL & $3.2(1.7-4.8)$ & $5.8(3.9-8)$ & 0.026 \\
\hline $\operatorname{ADMA}\left(10^{3} \mathrm{ng} / \mathrm{mL}\right)$ & $213 \pm 118$ & $386 \pm 297$ & 0.003 \\
\hline $\mathrm{WBC} 10^{3} / \mu \mathrm{L}$ & $5.8 \pm 2.5$ & $6.1 \pm 2.8$ & 0.595 \\
\hline Hemoglobin $(\mathrm{g} / \mathrm{dl})$ & $13.5 \pm 1.6$ & $12.7 \pm 1.6$ & 0.019 \\
\hline Neutrophil $10^{3} / \mu \mathrm{L}$ & $3.6 \pm 1.6$ & $3.9 \pm 1.8$ & 0.359 \\
\hline Eosinophil $* 1 / \mu \mathrm{L}$ & $60(20-187)$ & $10(20-70)$ & 0.033 \\
\hline Lymphocyte $10^{3} / \mu \mathrm{L}$ & $1.917 \pm 0.821$ & $1.429 \pm 0.674$ & 0.005 \\
\hline Monosite $10^{3} / \mu \mathrm{L}$ & $0.47 \pm 0.18$ & $0.38 \pm 0.20$ & 0.062 \\
\hline Favipiravir (n/\%) & $9(21.4)$ & $33(80.5)$ & $<0.001$ \\
\hline Oseltamivir (n/\%) & $25(59.5)$ & $24(58.5)$ & 0.928 \\
\hline Azithromycin (n/\%) & $24(57.1)$ & $27(65.9)$ & 0.421 \\
\hline Chloroquine (n/\%) & $39(92.9)$ & $32(78)$ & 0.056 \\
\hline Vitamin C (n/\%) & $2(4.8)$ & $18(43.9)$ & $<0.001$ \\
\hline $\begin{array}{l}\text { Dexamethasone (or potentially } \\
\text { other glucocorticoids) }(\mathrm{n} / \%)\end{array}$ & $1(2.4)$ & $7(17.1)$ & 0.023 \\
\hline Tocilizumab $(\mathrm{n} / \%)$ & $0(0)$ & $3(7.3)$ & 0.076 \\
\hline Convalescent plasma (n/\%) & $0(0)$ & $3(7.3)$ & 0.076 \\
\hline Enoxaparin (n/\%) & $18(42.9)$ & $30(73.2)$ & 0.005 \\
\hline
\end{tabular}

Continuous variables are given as mean $\pm S D$. BMI: body mass index. HT: Hypertension, HPL: Hyperlipidemia, DM: Diabetes Mellitus, BMI: Body Mass Index; SBP: Systolic Blood Pressure, DBP: Diastolic Blood Pressure, LVEF: Left Ventricular Ejection Fraction; CCB: Calcium Channel Blocker. ACEI: Angiotensin-converting enzyme inhibitors. ARB: Angiotensin II receptor blockers. ADMA: Asymmetric Dimethylarginine, WBC: White blood cell; CRP: C Reactive Protein. 
Table 2. Logistic regression analyze of ADMA with lung involvement and CRP.

\begin{tabular}{|l|c|c|c|c|c|c|}
\hline Parameters & \multicolumn{3}{|c|}{ Univariate } & \multicolumn{3}{c|}{ Multivariate } \\
\hline Variables & OR & $95 \%$ CI & $p$ & OR & $95 \%$ CI & $p$ \\
\hline Lung Inv. (+) & 3.333 & $1.284-8.653$ & 0.013 & 2.070 & $0.571-7.502$ & 0.268 \\
\hline CRP (Log) & 1.633 & $1.182-2.255$ & 0.003 & 1.633 & $1.182-2.255$ & 0.003 \\
\hline
\end{tabular}

ADMA: Asymmetric Dimethylarginine, CRP: C Reactive Protein.

had fever more frequently $(61.9 \%$ vs. 33.3 , $p=0.008$ ) compared to patients without lung involvement. The remaining characteristics including previous drugs and the presence of hypertension and coronary artery disease were not different between groups.

Patients with lung involvement had higher admission glucose (133.6 \pm 60 vs. 107.4 \pm 22.5 $m g / d l, \quad p=0.011)$, C-Reactive Protein (CRP) (3.85 \pm 1.22 vs. $1.72 \pm 1.39, \quad p<0.001)$, and ADMA concentrations $(386 \pm 297$ vs $213 \pm 118$, $p=0.003)$, and had lower hemoglobin concentration $(12.7 \pm 1.6 \mathrm{~g} / \mathrm{dl}$ vs. $13.5 \pm 1.6$, $p=0.019)$ and lymphocyte count $(1.429 \pm 0.674$ vs. $\left.1.917 \pm 0.82110^{3} / \mu l, p=0.005\right)$ compared to patients without lung involvement. Moreover, we observed a trend for decreased monocyte count in group 1, which did not reach statistical significance $(p=0.062)$. Interestingly, only 11 (13\%) patients had normal ADMA values (12.35 ng/mL and $1000 \mathrm{ng} / \mathrm{mL}), 90.5 \%$ of group 1, and $83.3 \%$ of group subjects had very high ADMA concentrations. We observed a good correlation between ADMA and CRP (Figure-1). As expected, patients in group 1 received favipiravir, vitamin $\mathrm{C}$, intravenous steroids, and anti-coagulants more frequently than the control subjects. Multivariate analysis including presence of fever, hemoglobin value, glucose concentration, lymphocyte count, and $\log$ ADMA identified log CRP concentration $(O R=3.047,95 \% C I=1.881-5.023, p<0.001)$ as the single independent predictor for lung involvement (Table 2).

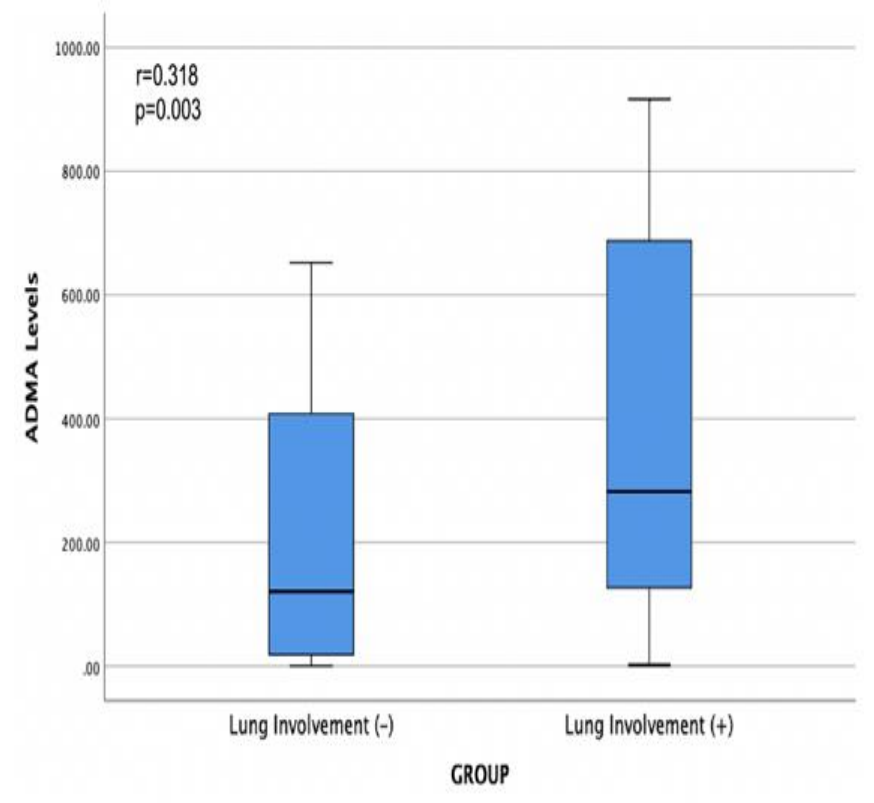

Figure 1. Relation of ADMA with lung involvement.

\section{Discussion}

The principal finding of this study is that the patients with lung involvement had higher ADMA concentrations with respect to those without lung involvement, plasma ADMA levels were also considerably higher than normal values in control group.

Vascular endothelium is an active paracrine, endocrine and autocrine organ invariably involved in regulation of vascular tonus and vascular homeostasis [5]. Autopsy series of deceased COVID-19 patients reported direct viral invasion of endothelial cells and widespread endothelial inflammation [8]. In addition to endothelial dysfunction, an imbalance towards vasoconstriction and prominent microvascular pro-coagulant activity is frequently encountered [9]. Correspondingly, 
Micro CLOTS is defined as a complication of severe COVID-19 infection with obstructive thrombo-inflammatory involvement of lung capillaries, which possibly reflects an atypical form of acute respiratory distress syndrome (ARDS) [10].

$\mathrm{NO}$ is a critical molecule that has a role in maintenance of organ perfusion and vascular balance $[11,12]$. ADMA is a protein breakdown product that inhibits endogenous NOS enzyme and thus lowers NO activity. A study involving critically ill patients in intensive care units revealed ADMA as the strongest independent predictor of mortality. Moreover, patients at the highest quartile had 17-fold increased mortality compared with the lowest tertile [13]. Therefore, ADMA accumulation has been proposed to be an etiologic factor that is involved in multiorgan failure pathogenesis due to decreased NO production [14].

In this study, the patients with lung involvement had higher ADMA concentrations, majority of the study patients had considerably higher plasma ADMA levels than normal values. We think that endothelial dysfunction is invariably present in COVID-19 disease whether there is an accompanying pneumonia or not. Interestingly, in the Cardiovascular Risk in Young Finns Study, investigators followed 1043 infection-related hospitalization in early childhood and documented adverse adulthood atherosclerotic changes 33 years later. Childhood infection-related hospitalization was closely associated with increased ADMA concentrations in the adulthood [15]. Although we don't have a long-term follow-up data regarding our patient population, we may hypothesize that elevated ADMA levels reflect inflammatory response severity in our study at the $1^{\text {st }}$ month of follow-up.

Inflammatory response has a central role with increasing severity of COVID-19 disease, especially when cytokine storm is present. CRP is a non-specific acute phase reactant synthesized by the liver, and a biological marker of inflammation, infection and tissue damage [15]. Computed tomography is the main imaging method to determine the severity of COVID-19 pneumonia. A recent study revealed increased CRP concentrations in the early stage of COVID-19 disease and CRP was an independent predictor of severe lung lesion [16]. Similarly, CRP in addition to interleukin 6 , was highly predictive of mechanical ventilation requirement [17]. We also identified CRP as the sole independent predictor of lung involvement similar to these studies.

Increased CRP activity may cause endothelial dysfunction due to inhibition of NO synthesis and bioactivity [18]. High ADMA levels were closely associated with CRP, cardiovascular risk factors, and cardiovascular death in patients with newly diagnosed diabetes mellitus [19]. Data suggests that ADMA and CRP are involved in endothelial dysfunction [20,21]. We identified CRP as the sole determinant of ADMA concentration and revealed a correlation between these parameters in our study, which are in accordance with the aforementioned studies.

Our study has several limitations that must be considered when interpreting these results, most importantly the small population size. We only have ADMA concentrations at the 1st month of follow-up after discharge. We lack serial ADMA measurement and a healthy control group. So, there is need for larger and multi-center studies which powered for improvement in clinical outcome (particularly thrombogenic events). Beyond these limitations, this is a pioneering study for further research that will focus on modalities to detect patients with pulmonary involvement at an early stage. 


\section{Conclusion}

The findings of this study emphasize that elevated ADMA concentrations as the surrogate of endothelial dysfunction in COVID-19 patients whether they have pneumonia or not. And, It deserves consideration as a laboratory parameter for our better understanding of COVID-19 physiopathology.

Funding: The author(s) received no financial support for the research, authorship, and/or publication of this article.

Conflict of Interest: The authors declare that they have no conflict of interest.

\section{Ethical statement:}

The study was approved by Local Clinical Research Ethics Committee (Date and Decision Number: 2020/164), and written informed consent was obtained from each subject.

\section{Open Access Statement}

This is an open access journal which means that all content is freely available without charge to the user or his/her institution under the terms of the Creative Commons Attribution NonCommercial License

(http://creativecommons.org/licenses/bync/4.0). Users are allowed to read, download, copy, distribute, print, search, or link to the full texts of the articles, without asking prior permission from the publisher or the author.

Copyright (c) 2021: Author (s).

\section{References}

[1]Park SE. Epidemiology, virology, and clinical features of severe acute respiratory syndrome -coronavirus-2 (SARS-CoV-2; Coronavirus Disease-19). Clin Exp Pediatr. 2020;63(4):119-24.

[2]World Health organization; WCDCCoronavirus Disease (COVID-19) Dashboard [updated 05/09/2020.
[3]Wu Z, McGoogan JM. Characteristics of and important lessons from the coronavirus disease 2019 (COVID-19) outbreak in China: summary of a report of 72314 cases from the Chinese Center for Disease Control and Prevention. JAMA. 2020;(323):123942.

[4]Ferrario CM, Jessup J, Chappell MC, et al. Effect of angiotensin-converting enzyme inhibition and angiotensin II receptor blockers on cardiac angiotensin-converting enzyme 2. Circulation. 2005;(111):2605-10.

[5]Flammer AJ, Anderson T, Celermajer DS, et al. The assessment of endothelial function: from research into clinical practice. Circulation. 2012;(126):753-67.

[6]Cekmen M, Turgut M, Turkoz Y, et al. Nitric Oxide and Nitric Oxide Synthaze Physiologic and Pathologic Characterictics. T Klin J Ped. 2001;(10):226-35.

[7]Raghavan SA, Dikshit M. Vascular regulation by the L-arginine metabolites, nitric oxide and agmatine. Pharmacol Res. 2004;(49):397-14.

[8]Varga Z, Flammer AJ, Steiger P, et al. Endothelial cell infection and endotheliitis in COVID-19. The Lancet. 2020;(395):141718.

[9]Bonetti PO, Lerman LO, Lerman A. Endothelial dysfunction: a marker of atherosclerotic risk. Arterioscler Thromb Vasc Biol. 2003;23(2):168-75.

[10]Ciceri F, Beretta L, Scandroglio AM, et al Microvascular COVID-19 lung vessels obstructive thromboinflammatory syndrome (MicroCLOTS): an atypical acute respiratory distress syndrome working hypothesis. Crit Care Resusc. 2020;22(2):95-97.

[11]Cooke JP. Derangements of the nitric oxide synthase pathway, L-arginine, and 
cardiovascular diseases. Circulation. 1997;(96):379-82.

[12] Napoli C, Ignarro LJ. Nitric oxide and atherosclerosis. Nitric Oxide. 2001;(5):8897.

[13] Nijveldt RJ, Teerlink T, Van Der Hoven B, Siroen MP, Kuik DJ, Rauwerda JA, van Leeuwen PA. Asymmetrical dimethylarginine (ADMA) in critically ill patients: high plasma ADMA concentration is an independent risk factor of ICU mortality. Clin Nutr. 2003;22(1):23-30.

[14] Nijveldt RJ, Teerlink T, van Leeuwen PA. The asymmetrical dimethylarginine (ADMA)-multiple organ failure hypothesis. Clin Nutr. 2003;22(1):99-104.

[15]Burgner DP, Sabin MA, Magnussen CG, et al. Early childhood hospitalisation with infection and subclinical atherosclerosis in adulthood: the Cardiovascular Risk in Young Finns Study. Atherosclerosis. 2015;(239):496-502.

[16]Ling W. C-reactive protein levels in the early stage of COVID-19. Medecine et Maladies Infectieuses. 2020;50(4):332-34.

[17] Herold T, Jurinovic V, Arnreich C, et al. Elevated levels of IL- 6 and CRP predict the need for mechanical ventilation in COVID19. J Allergy Clin Immunol. 2020;146(1):128-136.e4.

[18] Singh U, Devaraj S, Vasquez-Vivar J, et al. C-reactive protein decreases endothelial nitric oxide synthase activity via uncoupling. J Mol Cell Cardiol. 2007;(43):780-91.

[19] Triches CB, Mayer S, Quinto BMR, et al. Association of endothelial dysfunction with cardiovascular risk factors and new-onset diabetes mellitus in patients with hypertension. J Clin Hypertens. 2018;(20):935-41.
[20]Cooke JP. Does ADMA cause endothelial dysfunction? Arterioscler Thromb Vasc Biol. 2000;20(9):2032-37.

[21]Baylis C. Arginine, arginine analogs and nitric oxide production in chronic kidney disease. Nat Clin Pract Nephrol. 2006;2(4):209-20. 